\section{Journal of Radiotherapy in Practice}

\title{
Abstracts: Radiotherapy in Practice Conference, Sheffield, September 2003
}

\author{
Programme - Radiotherapy in practice conference
}

\section{SATURDAY 27TH SEPTEMBER 2003}

$9.15-9.55$
$10.00-10.10$
$10.10-10.50$
$10.50-11.20$
$11.20-11.30$
$11.30-12.00$
$12.00-12.15$
$12.15-12.30$
$12.30-12.45$
$12.45-1.00$
$1.00-2.00$
$2.00-2.40$

$2.40-4.00$

$2.40-2.50$

$2.50-3.00$
Conference registration

Welcome and opening address

Professor Rhiannon Billingsley (SHU)

Modernising the cancer workforce Carol Wilby (DoH)

Modernising radiotherapy education and professional development Angela Duxbury, David Eddy \&

Angela Eddy (SHU)

Open forum

Coffee/exhibition

Workforce planning update Martine Jackson (Manchester)

Are extended working days sustainable? Donna Routsis (Cambridge)

Future of the radiotherapy workforce Stephanie McWilliam (DoH)

Open forum/questions to panel

Lunch and exhibition

Medico-legal and ethical dilemmas for healthcare practitioners

Dr David Radstone (Sheffield)

Parallel sessions

1. Counselling skills workshop Denyse Hodgson (SHU)

2. Informed consent case studies Angela Eddy (SHU)

3. Reflective practice workshop David Eddy (SHU)

4. Proffered papers and open forum Chair: Rob Appleyard (SHU)

\section{Proffered papers session}

Physical symptoms experienced by women receiving radiotherapy for breast cancer: the women's perspective Angela Cross (Univ. of Liverpool)

Prevalence, risk and severity of sexual dysfunction in pre-menopausal women having surgery or radiotherapy for cervical cancer

Maria Murray (Glasgow Caledonian University)

\begin{tabular}{ll}
\hline $3.00-3.10$ & $\begin{array}{l}\text { Read my my lips! How to read more } \\
\text { effectively } \\
\text { Louise Waywell (Univ. of Liverpool) }\end{array}$ \\
$3.10-3.20$ & $\begin{array}{l}\text { Implementing a local imaging protocol with a } \\
\text { national perspective } \\
\text { Angela Heaton (Liverpool) }\end{array}$ \\
$3.20-3.30$ & $\begin{array}{l}\text { A review of portal imaging strategies for } \\
\text { radical lung patients at Rosemere Cancer }\end{array}$ \\
& $\begin{array}{l}\text { Centre } \\
\text { Darran Kellett (Preston) }\end{array}$ \\
$3.30-3.40$ & $\begin{array}{l}\text { Assessment of optimal fiducial marker length } \\
\text { for pelvic radiotherapy verification }\end{array}$ \\
$3.40-4.00$ & $\begin{array}{l}\text { Julie Stratford (Manchester) } \\
\text { Open forum }\end{array}$ \\
$4.00-4.30$ & Coffee and exhibition \\
$4.30-4.50$ & Service improvement initiatives \\
& Angie Craig (Cancer Services Collaborative) \\
$4.50-5.30$ & Future directions for radiotherapy \\
Dr DV Ash (Leeds) & Open forum \\
$5.30-5.40$ & Social event \\
Evening &
\end{tabular}

SUNDAY 28TH SEPTEMBER 2003

\begin{tabular}{ll}
\hline $9.30-9.40$ & $\begin{array}{l}\text { Welcome back and hello new delegates } \\
\text { Dr Linda Lang (SHU) }\end{array}$ \\
$9.40-10.20$ & $\begin{array}{l}\text { Future of radiotherapy imaging } \\
\text { Dr Andy Beavis (Hull) }\end{array}$ \\
$10.20-10.40$ & $\begin{array}{l}\text { WPH imaging project } \\
\text { Dr Mark McJury (Sheffield) }\end{array}$ \\
$10.40-11.00$ & $\begin{array}{l}\text { Radiotherapy treatment of prostate cancer } \\
\text { using high dose rate brachytherapy boost }\end{array}$ \\
Dr Heather Payne (Middlesex) \\
$11.00-11.30$ & $\begin{array}{l}\text { Coffee } \\
11.30-11.45\end{array}$ \\
$\begin{array}{l}\text { Learning opportunities in organisational } \\
\text { change }\end{array}$ \\
$\begin{array}{l}\text { Joy Brumby (Royal Free) } \\
\text { A study of radiotherapy workload and } \\
\text { staffing using the basic treatment equivalent } \\
\text { Sue Griffiths (Leeds) }\end{array}$ \\
\hline
\end{tabular}




\begin{tabular}{|c|c|}
\hline $12.15-12.30$ & $\begin{array}{l}\text { "...and a Partridge in a Pear Tree" } \\
\text { Managing clinical training burden in a modern } \\
\text { radiotherapy department } \\
\text { Margaret Abraham (Preston) }\end{array}$ \\
\hline $12.30-12.40$ & Open forum \\
\hline $12.40-1.40$ & Lunch and exhibition \\
\hline $1.40-3.10$ & $\begin{array}{l}\text { Parallel sessions } \\
\text { 1. Critical thinking and writing } \\
\text { Dr Heidi Probst (SHU) } \\
\text { 2. Portfolio clinic } \\
\text { David Eddy (SHU) } \\
\text { 3. Mapping services and initiating change } \\
\text { Angie Craig (CSC) } \\
\text { 4. Proffered papers and open forum } \\
\text { Chair: Angie Eddy (SHU) }\end{array}$ \\
\hline \multicolumn{2}{|c|}{ Proffered papers session } \\
\hline $1.40-1.50$ & $\begin{array}{l}\text { Real-time point tracking for radiotherapy } \\
\text { patients using stereoscopy } \\
\text { Christine Rawlings (Torbay) }\end{array}$ \\
\hline $1.50-2.00$ & $\begin{array}{l}\text { The efficiency and effectiveness of auto-field } \\
\text { sequencing applied during radiotherapy } \\
\text { Jillian Moses (Aberdeen) }\end{array}$ \\
\hline
\end{tabular}

$2.10-2.20$

$2.20-2.30$

$2.40-2.50$

$2.50-3.00$

$3.00-3.10$

$3.10-3.30$

$3.30-3.50$

$3.50-4.00$
Improving the accuracy in delivery of IMRT using on-line verification Julie Davies (Manchester)

Minimising tumour movement in lung radiotherapy with an automatic breathing control device: initial findings Bridget Porritt (Manchester)

Prostate set-up best practice development MRC RT01 trial Sue Griffiths (Leeds)

Simple cardiac shielding in breast radiotherapy Claire McCarthy (Manchester)

Open forum

Coffee

Conference plenary-thoughts for the future Angela Duxbury (SHU)

Awards and closing remarks David Eddy (SHU) 


\section{'Radiotherapy in Practice' Abstracts}

\section{The efficiency and effectiveness of auto field sequencing applied during radiotherapy treatment}

J. Moses, Jo Child

Grampian University Hospital Trust

The aim of the study is to investigate the potential benefits from the use of auto field sequencing (AFS) during everyday treatment. The main objectives for this study will be to examine patient satisfaction, the effectiveness of patient throughput, quality and accuracy of treatment and patient satisfaction.

During this study we will recruit a sample of 40 radical pelvis patients, who will be split into two distinct groups. The patients are recruited at time of booking and are split into groups dependent upon the treatment machine where treatment will take place. The first group will receive their treatment using AFS, which will be carried out by an individual treatment machine, and the second group will receive their treatment with the manual rotation of the machine into the next treatment position, which will be carried out, by another machine. These treatments will be monitored using the treatment times recorded by the record and verify computer. The patients in the two arms of the study will have their times monitored and compared with each other, to see the affect AFS has on treatment time.

Quality of treatment will be monitored using electronic portal imaging connected to the treatment machine. The portal images will then be compared using the simulator image as gold standard and reviewed using anatomical matching software. Images will be taken once a week during the patient's treatment. A comparison between the two group's images will take place involving the monitoring of ant/post movement, sup/inf movement and lateral movement.

Patient satisfaction analysis will be used to help monitor patient satisfaction. An identical question- naire will be distributed to all patients to identify the differences in answers between the two groups.

From the results of this study the findings we anticipate are reduced treatment times and no alteration in treatment quality and patients satisfaction. These anticipated findings are based on a pilot study which identified the need for further research.

The findings will help in the production of protocols for the use and safety procedures needed during the use of AFS.

\section{Physical symptoms experienced by women receiving radiotherapy for breast cancer: the women's perspective}

\section{A. Cross}

\section{Division of Radiotherapy, University of Liverpool}

Breast cancer is a common malignancy found in women and is frequently treated by breast conserving surgery and radical radiotherapy. Radiotherapy is likely to produce a range of physical symptoms and patients need to be informed about these symptoms and their effects. In order to determine these physical symptoms two studies were undertaken. The first, a qualitative study, examined 15 women's own accounts of physical symptoms during the first 12 months after treatment. The women described many symptoms and their explanations for the symptoms were an inextricable component of their experience of them. The second study was a quantitative study using the Physical Experiences Questionnaire developed from the findings of Study 1 . The aims of Study 2 were to quantify the physical symptoms and to determine change over time. In addition patients attributions for the symptoms at different times were examined. Finally, patients' fatigue, anxiety and depression were examined to find out whether they were related to symptoms. 
The findings showed patients experienced a wide range of symptoms. The diversity of attributions for symptoms indicated patients' need to make sense of bodily experiences.

This study provides an important insight into the women's perceptions of their experiences of radiotherapy. This paper will discuss the two studies, highlighting the main findings and recommendations.

\section{Read my my lips! How to read more effectively}

L. Waywell, G. Smith

\section{Radiotherapy Division, University of Liverpool}

Undergraduates often find difficulty adjusting to the different demands expected in higher education. An expected requirement of any course is the large volume of material to read. The Radiotherapy Division at The University of Liverpool felt it would benefit students if it could assist with the acquisition of additional skills, such as speed reading.

Most people are slow inefficient readers with an average reading speed of 200-250 words/minute. After learning to read there is almost a complete lack of further instruction, resulting in adults being less efficient readers than they could be. Reading should be considered similar to other skills where constant practise is required to improve.

Increasing reading efficiency allows individuals greater absorption, integration and retention of knowledge. By doubling reading efficiency, twice as much information can be read in the same time period. Care needs to be taken to ensure that increases in speed are not detrimental to understanding and recall.

The Radiotherapy Division developed a 2-day intensive reading course, with the aim of doubling participants reading speed. The course contains a variety of different exercises encouraging reading habits to change. Day one concentrates on eye speed using the Reading Accelerator ${ }^{\circledR}$, a lightweight hand held training instrument, allowing manipulation of reading pace. This helps to increase total reading ability via improvement of reading rate, efficiency, fluency and flexibility. Day two concentrates on comprehension and retention of information.

This course has been run successfully over the last 6 years for our undergraduates and the University staff development programme.

\section{Prevalence, risk and severity of sexual dysfunction in pre-menopausal women having surgery or radiotherapy for cervical cancer}

M. Murray

Glasgow Caledonian University, Glasgow

Purpose: To assess prevalence, risk and severity of sexual dysfunction in pre-menopausal women having surgery or radiotherapy for cervical cancer. To investigate sexual function information needs.

Patients: Seventy nine pre-menopausal women, from the West of Scotland, with Stages I, II, or III cervical cancer were invited and with the rigourous exclusion/inclusion criteria, 48 participated.

Methods: A prospective, longitudinal and investigative study. Assessment involved validated self-report instruments for sexual dysfunction and anxiety and depression with measures of vaginal stenosis, over the period of one year following either surgery $(n=24)$ or radiotherapy $(n=24)$ and included a one year pre-morbid retrospective measure. A small qualitative study assessed information needs. Descriptive and inferential statistics included the use of Chi-Square, $\mathrm{McNemar}$ and Wilcoxon Signed Ranks Tests.

Results: Both surgery and radiotherapy subjects had levels of sexual dysfunction following treatment. Vaginal stenosis appeared in $88 \%$ of radiotherapy subjects. Higher percentages of surgery subjects developed sexual dysfunctional levels with greater decreases in sexual frequency. Sexual dissatisfaction was minimally affected. Surgery subjects $(73.6 \%)$ were more likely to avoid sexual activity one year post treatment than radiotherapy subjects (47\%). Surgery subjects developed sexual difficulty 4 months post treatment (20\%) which decreased, at one year after $(10.5 \%)$, when radiotherapy subjects had higher levels (23.5\%). Anxiety was a problem for the surgery group. A number of subjects were 
given no sexual advice/information and for those who were, it was generally helpful and reassuring.

Conclusions: Cervical cancer treatment, anxiety levels and stenosis contribute to sexual function problems in the pre-menopausal woman, whether the treatment is surgery or radiotherapy. Information booklets, specifically regarding sexual function, should be written for patients and partners. Patients would like more advice and information from female health professionals with their partner present.

\section{Simple cardiac shielding in breast radiotherapy}

C. McCarthy, J. Davies, B. Magee

Wade Centre for Radiotherapy Research, Christie Hospital, Manchester

Aim: At this institute we conducted a retrospective analysis to determine whether shielding the cardiac tissue using a single MLC leaf would significantly reduce the given dose to the heart.

Methodology: The single slice CT data of ten patients was loaded onto the planning system (ADAC), areas of interest such as the original treatment volume, and the heart, were contoured, and using the patient's original treatment data (field size, wedge, TD etc.) a treatment plan was produced. We then produced a second plan using the same treatment data, which included a single MLC along the wedge direction to shield the heart.

Results: The dose volume histograms for each plan were compared. This comparison showed that by using a single MLC we could significantly reduce the volume of heart irradiated. It also showed a significant reduction in the volume of the original treatment area receiving the required treatment dose.

Conclusions: This work demonstrates that the ideal progression for breast radiotherapy would be to develop a conformal or IMRT based treatment technique, which would allow for a reduction in the volume of cardiac tissue treated without compromising on the therapeutic dose delivered to the breast.

\section{Minimising tumour movement in lung radiotherapy with an automatic breathing control device: initial findings}

\author{
B. Porritt, J. Davies, B. Perrin, P. Burt, R. Stout \\ Wade Centre for Radiotherapy Research, Christie \\ Hospital, Manchester
}

Aim: To investigate the suitability of an Active Breathing Co-Coordinator ${ }^{\mathrm{TM}}$ (ABC), Elekta Oncology Systems, in reducing tumour motion in patients receiving radiotherapy for carcinoma of the lung.

Method: This is a feasibility study, recruiting patients with non-small cell carcinoma of the lung, being treated with radical intent. A short training session takes place prior to simulation, to ensure participants can comfortably and consistently reproduce breath holds. Patients are simulated and CT scanned with the ABC device, after the conventional simulation and radiotherapy planning scan. The fluoroscopy and CT data acquired is analysed to compare tumour movement observed with and without the use of the ABC device.

Results: 4 patients have completed planning sessions. All patients tolerated the $\mathrm{ABC}$ device well and achieved a 15 to 20 second breath hold for 5 to 8 repeated holds. Data acquired shows that tumour motion is reduced with the use of the $A B C$ device.

Conclusion: This early experience suggests that $\mathrm{ABC}$ is tolerable and reproducible. Tumour movement is reduced; the question of whether this will offer the possibility of reducing planning margins and allow escalation of dose is still to be answered.

\section{Assessment of optimal fiducial marker length for pelvic radiotherapy verification}

J. Stratford, J. Davies, K. Bone, J. Sykes, C. Moore, J. Logue, V. Khoo

Academic Department Clinical Oncology, Christie Hospital, Manchester

Aim: To assess optimal length of fiducials for treatment setup/verification in pelvic radiotherapy and to evaluate factors influencing visualisation. 
Methods: Gold fiducials of $8,5,4$ and $3 \mathrm{~mm}$ (all with 0.8 diameters) were placed on entry and exit sites (AP and lateral directions) in a group of 18 patients. A template of 14 fiducials for each size was used.Visualisation from an amorphous silicon panel was assessed using subjective assessment scale by 3 independent observers. Results are presented.

Results: Total visibility for each seed size 8, 5, 4 and $3 \mathrm{~mm}$ incorporating all views and all observers were $97.4 \%, 90.6 \%, 93.5 \%$ and $84.3 \%$ respectively. Qualitative assessment revealed that overlap of bony structures in the lateral views provided more difficult visualisation compared to the AP direction.Visibility was associated with fiducial marker length. $8 \mathrm{~mm}$ was significantly better $(\mathrm{p}<0.0005)$ than 5,4 and $3 \mathrm{~mm}$. There was no significant difference in visibility between $4 \mathrm{~mm}$ and $5 \mathrm{~mm} .3 \mathrm{~mm}$ length was significantly inferior to 8,5 and $4 \mathrm{~mm}(\mathrm{p}<0.0005)$.

Conclusion: Optimal length of fiducial marker for treatment setup/verification was found to be $8 \mathrm{~mm}$. This will be further tested in a feasibility study of implantation for patients undergoing pelvic radiotherapy.

\section{Improving the accuracy in delivery of IMRT using on-line verification}

\author{
J. Davies, J. Stratford, C. McCarthy, B. Porritt, \\ J. Livsey, J. Logue \\ Radiotherapy Research, Christie Hospital, Manchester
}

Aim: To verify that on-line verification and active intervention, can ensure for IMRT, treatment tolerances of $3 \mathrm{~mm}$ and below.

Methods: Prostate cancer patients treated with IMRT were imaged on-line, days 1 to 5 and weekly thereafter using I-View GT (ELEKTA). Pretreatment corrections were performed where necessary. Data from 102 image pairs were analysed to evaluate frequency of intervention. Post treatment verification was also analysed to identify intra-fraction movement.

Results: In twenty four percent of cases pretreatment intervention was required to ensure tolerances remained within $3 \mathrm{~mm}$, (2.9\% to remain within $5 \mathrm{~mm}$ ) intra-fraction median movement was $0.35 \mathrm{~mm}$ (range of $0-4.5 \mathrm{~mm}$ ).

Conclusion: This study verified that the accuracy of IMRT delivery could be improved by implementing a policy of on-line verification. This accuracy in positioning could be maintained throughout a treatment fraction.

\section{Implementing a local imaging protocol and a national perspective}

\section{A. Heaton}

\section{Clatterbridge Centre for Oncology, Wirral}

This presentation will consider the current provision of imaging equipment and protocols in the UK. It will give results of a national questionnaire which asked details of pre-treatment and ontreatment imaging. Results are presented showing slice intervals used for CT Planning, use of DRR's, frequency of portal imaging, tolerances applied and a discussion of responsibilities for image analysis.

The implementation of a local imaging protocol into a department will be considered next. This will discuss the approach taken at Clatterbridge and will cover the formulation and objectives of a radiographer-led imaging group and training requirements for staff. Details will then be given of the current clinical protocol including why it was decided not to differentiate between radical and palliative treatments and to image all isocentric arrangements and all patients with beam shaping, the frequency of imaging, systematic and random errors, action levels used and the assignment of responsibilities.

The next section will discuss lessons learned and the need for comprehensive guidelines to support practice and the need for audit to develop and re-evaluate working practices to improve the use of portal imaging in radiotherapy.

The presentation will conclude with some current and future development ideas happening at Clatterbridge including IMRT imaging and the use of portal imaging dosimetry, soft tissue 
movement analysis, site specific protocols and reassigning responsibilities to give role-extension to radiographers.

\section{Learning opportunities in organisational change}

J. Brumby

Royal Free Hospital, Hampstead, London

The Peter MacCallum Cancer Centre is a large specialist cancer treatment hospital in Melbourne, Australia. The centre comprises a main campus with 5 linear accelerators, three satellite centres with 2 linear accelerators each and a single machine rural site. The Centre employs over 120 radiation therapists, and provides services in all clinical radiation oncology areas, including specialties such as intensity modulated radiation therapy (IMRT), stereotactic radiosurgery, prostate seed implants, total body irradiation (TBI) and paediatric radiotherapy. In February 1998 , a process redesign project was initiated at the main campus of the Peter MacCallum Cancer Centre. The project focused on the radiotherapy treatment planning process. The objective was to streamline the planning process to ensure efficient utilization of resources and compliance with defined clinical indicator guidelines for the interval between the initiation of treatment planning and the commencement of treatment. In redesigning the planning process, all radiation therapists involved learned from the process in the areas of workload management, team formation and targeted areas for technical training. Since that time, the teaching, developmental and learning opportunities have continued for all radiation therapists in the department, who are rostered on a rotational basis between planning and treating. This paper discusses the educational gains which have occurred as a result of the functional and cultural change across the department, and the issues which arise. One of the key factors influencing the successful expansion of the model has been the implementation of satisfactory educational strategies, as educational support is critical to the success of the redesign. All organisational change presents learning opportunities, and in today's climate of accountability and the shift towards mandatory continuous professional development (CPD), it is important to recognize these opportunities and maximise the potential for professional growth of the individuals involved.

\section{A review of portal imaging strategies for radical lung patients at Rosemere Cancer Centre}

D. P. Kellett, M. C. Kirby

\section{Radiotherapy Department, Rosemere Cancer Centre, Royal Preston Hospital}

Portal Imaging is a fundamental and wellestablished element of the Radiotherapy process within the Rosemere Cancer Centre. However, it is part of Rosemere policy to review all radiotherapy procedures for all patients on a regular basis, using a multidisciplinary approach. The review of portal imaging strategies has a number of on-going benefits, not least of which is possible improvements of image quality and reductions in the number of repeated films and images. This paper will describe our current audit of practice for radical lung patients.

The key subsections to be examined in this work are:

1. Current practice - review image quality currently obtained from both electronic portal imaging devices (Elekta iView and iViewGT (aSi) EPIDs), examine acquisition protocols, dose to normal tissues from double exposures etc. and frequency of repeat images.

2. Image quality comparisons - anthropomorphic phantom based studies for all imaging modalities.

3. Changes to clinical protocols - review frequency of imaging for radical patients and compare with other strategies, examine the possibility of a simpler imaging protocol with better defined dose recording.

4. Possible changes in field placement error analysis - determine the effectiveness and robustness of new analysis software available on the iViewGT (aSi) systems and compare with "gold standard" software (PIPSpro).

5. Clinical implementation - establish a new protocol based on current findings and implement on a small cohort of patients. 
Our initial results on image quality comparisons show qualitatively the superior imaging capability of the aSi EPIDs and how the camera based EPID image quality may be improved by using single open fields instead of double exposures. Current clinical protocols have been reviewed and a strategy for orthogonal, open field imaging, with welldefined dose limits, has been approved. The new iViewGT analysis software has been commissioned successfully, and a reticule-based protocol is being piloted for IMRT patients.

On completion of the review a modified, evidence based, clinical protocol will be put in place, and the successful protocol review process will be applied to other anatomical sites.

\section{Real time point tracking for radio- therapy patients using stereoscopy \\ C. Rawlings \\ Torbay Hospital, Torbay}

Aim: A real time point tracking system has been developed to monitor patient positioning during radiotherapy and to formalise tolerance levels for treatment set-up.

Materials and method: The system has been adapted from the commercially available Osiris outlining device and incorporates stereo mathematics. Patients' skin marks can be tracked without the use of special markers. Rapid camera switching enables an average of 50 data points per camera to be collected for each field. Portal films are used to compare bony anatomy with the skin surface data, in bladder and prostate patients. Positional and isocentric errors are displayed during treatment and stored for individual patients. Prostate, breast and bladder patients have been monitored and have demonstrated varying degrees of positional errors.

Results: In the prostate patients positional errors ranged from $3 \mathrm{~mm}$ in a moving patient to below $1 \mathrm{~mm}$ for a stable patient. Good immobilisation has been shown with the breast patients and the system has also demonstrated shape changes in these patients. Bladder patients receiving treatment, within the BC2001 trial have been monitored and comparison made with portal films. Time trend data has also been accrued demonstrating first fraction and entire course movement. Portal film analysis shows correlation with the point tracking system, but errors outside the tolerance levels set have been recorded. This may be as a result of particular patient characteristics, such as obesity.

Conclusion: The system is both reliable and accurate in detecting positional errors. It will enable confident auto sequencing and it is hoped that it will lead to gating of the Linear Accelerator if tolerance levels are exceeded.

\section{MRC RT01 trial (ISRTCTN 47772397) quality assurance and radiographer trial implementation group: prostate set-up best practice development}

\section{S. Griffiths, S. Stanley, L. Cassapi, and RT01 \\ Radiographer Trial Implementation Group on behalf of all the RT01 Collaborators}

Intentions: The RT01 trial, comparing standard with high dose conformal radiotherapy, required treatment delivery to within $5 \mathrm{~mm}$ in 3 dimensions. The quality assurance group devised comprehensive QA guidelines, assessing centres before and during the trial. Radiographer expertise was critical so the Radiographer Trial Implementation Group, including radiographers from all UK participating centres met to:

- Implement radiographer-led portal imaging, measurement and field correction, and ensure appropriate data collection.

- Monitor technique accuracy and develop best practice methods for the radiotherapy process.

- Share and learn from proven good practice.

Results: $80 \%$ of participating centres made revisions to practice during the trial: patient positioning 55\%; isocentre setting methods 45\%; couch top $45 \%$, and progress towards evidencebased consensus on the detail of set-up methods was achieved. 
Conclusions: The Radiographer Trial Implementation Group, as part of the trial QA process, has enabled evidence-based progress towards best practice for conformal prostate setup. Portal image analysis expertise was developed and is accepted practice within the group. The expertise gained enabled CFRT to become routine practice in participating centres and is available for further trials.

\section{Introduction to the Radiotherapy Imaging Centre at Weston Park Hospital}

\author{
R. H. Ireland, M. McJury
}

Department of Radiotherapy Physics, Weston Park Hospital, Sheffield Teaching Hospitals, Sheffield, UK

High quality medical imaging is the key to improving radiotherapy treatment. At Weston Park Hospital, the introduction of the Radiotherapy Imaging Centre has led to many developments in the way that images are used in the treatment of patients with radiotherapy.

The developments have included:

- Installation of an advanced multileaf collimator suitable for IMRT.

- Installation of the Varian Medical Systems Varis Vision network of computer workstations for the acquisition, manipulation and review of radiotherapy images.

- Improvements to the availability and use of diagnostic images (including CT, MRI and PET) for treatment planning.

- Improvements to simulator images through the use of distortion corrected digital images with the option for large field of view images.

- The introduction of amorphous silicon portal imaging systems for improved treatment images that are used to assess the accuracy of the delivered treatment.

- The provision of networked access to radiotherapy images for on-line review and approval.

In summary, the Radiotherapy Imaging Centre provides better quality radiotherapy images, which has led to improvements in treatment planning and in the assessment of treatment accuracy. It is now possible for the digital images to be accessed simply and quickly whenever and wherever they are required for review. Along with the installation of multileaf collimators, the Radiotherapy Imaging Centre has provided Weston Park Hospital with the imaging tools required to introduce the technique of intensity modulated radiotherapy (IMRT).

The Radiotherapy Imaging Centre was established with $\mathcal{E}^{483,448}$ from the Weston Park Hospital Cancer Appeal, the largest grant ever awarded by the charity. 